\title{
Nonlinear Stabilizers in Optimal Control Problems with Infinite Time Horizon
}

\author{
Alexander Tarasyev and Anastasia Usova ${ }^{\star}$ \\ Institute of Mathematics and Mechanics \\ of the Ural Branch of the Russian Academy of Sciences, \\ S.Kovalevskaja Str. 16, 620990, Ekaterinburg, Russia \\ tam@imm.uran.ru, tarasiev@iiasa.ac.at, \\ anastasy.ousova@gmail.com \\ http://www.imm.uran.ru/engl.asp
}

\begin{abstract}
In optimal control problems with infinite time horizon, arising in models of economic growth, there are essential difficulties in analytical and even in numerical construction of solutions of Hamiltonian systems. The problem is in stiff properties of differential equations of the maximum principle and in non-stable character of equilibrium points connected with corresponding transversality conditions. However, if a steady state exists and meets several conditions of regularity then it is possible to construct a nonlinear stabilizer for the Hamiltonian system. This stabilizer inherits properties of the maximum principle, generates a nonlinear system with excluded adjoint variables and leads its trajectories to the steady state. Basing on the qualitative theory of differential equations, it is possible to prove that trajectories generated by the nonlinear stabilizer are close to solutions of the original Hamiltonian system, at least locally, in a neighborhood of the steady state. This analysis allows to create stable algorithms for construction of optimal solutions.
\end{abstract}

Keywords: optimal control, nonlinear control system, nonlinear stabilizer, economic systems.

\section{Introduction}

This paper deals with optimal control problems with infinite time horizon basing on economical growth models which is relied on classical constructions of growth theory (see [10], 11]). Also it includes ideas of a SEDIM model [9] describing

\footnotetext{
* The research is supported by the Russian Fund of Basic Research (Grants 11-010042-a, 11-01-12088-ofi-m-2011, 11-01-12112-ofi-m-2011), by the Program for the Sponsorship of Leading Scientific Schools (Grant NSCH-64508.2010.1), by the Program of the Presidium of RAS "Dynamic Systems and Control Theory", by the Program of the Presidium of RAS No. $38 \Pi$ (Project 12- $\Pi-1-1038$ ), by the Project of the Ural Branch of RAS "Socio-Economic Development of Regions: Forecasting and Optimal Control" (Grant 12- $\Pi-7-1001)$, and the International Institute for Applied System Analysis (IIASA).
}

D. Hömberg and F. Tröltzsch (Eds.): CSMO 2011, IFIP AICT 391, pp. 286-295, 2013.

(C) IFIP International Federation for Information Processing 2013 
the role of different economic factors such as the demographic ones in a country's economic development. Another technique in the background (see [2, [5]) considers capital and useful work as the key drivers of economic growth and uses optimal control theory to design past and future growth trajectories.

The research of optimal control problems uses as basis the Pontryagin's maximum principle [8] for the problem with infinite time horizon (see [1], [3, [5]). We investigate properties of the maximized Hamiltonian function and provide analysis of existence of steady states in domains of specific control regimes and focus attention on the domain corresponding to the transient control regimes of investment. We consider linearized Hamiltonian system in this domain. Special attention is given to the Jacobi matrix which has two negative and two positive eigenvalues that is the steady state has the saddle character. According to the results of the qualitative theory of differential equations [4] the trajectory of the nonlinear Hamiltonian dynamics converges to the steady state tangentially to the plane generated by eigenvectors corresponding to negative eigenvalues of the Jacobi matrix. This analysis provides the important information about the growth rates of optimal synthetic trajectories.

A novelty of the proposed solution is based on the idea of creating of nonlinear stabilizers built on the feedback principle (see [6], [7]) which lead the system from any current position to a steady state. The constructed nonlinear stabilizer generates the dynamic system closed in phase variables and having the property of local stability. Also we construct solutions of the Hamiltonian system and the stabilized Hamiltonian system in a steady state neighborhood and compare behavior of these trajectories. Simulated optimal trajectories of nonlinear Hamiltonian systems are obtained numerically by the implicit Runge-Kutta method.

\section{Two-Sectors Economical Growth Model and Optimal Control Problem}

The Model. The model is based on analysis of the Gross Domestic Product (GDP) dynamics which is denoted by symbol $Y$. It is supposed that changes of GDP depend on three production factors: capital stock $K$, labor $L$ (or it can be named as human capital) and useful work $U$. The production function $F$ describes the relation between these factors and GDP $(Y)$, that is $Y=F[K, L, U]$. It is assumed that the production function $F$ has the property of homogeneity of degree one, i.e.

$$
F[\alpha K, \alpha L, \alpha U]=\alpha F[K, L, U] \quad \forall \alpha>0 .
$$

This model includes also a parameter $P(t)$ denoting the number of workers in a country at time $t$. According to the Sanderson model [9] we assume that labor $L$ is proportional to the number of workers $P$ with coefficient $E$. This coefficient has the sense of labor efficiency of one worker. Hence, we have the following equality: $L(t)=E(t) P(t)$. Due to the homogeneous property of the production function we introduce relative variables: $k=K / P, l=E=L / P, u=U / P, y=Y / P$. 
It is supposed that the total number of workers $P$ has exponential growth trend

$$
\dot{P}(t)=\rho P(t), \quad \rho>0 .
$$

Here $\rho$ is a positive predefined constant denoting the relative growth rate. It should be mentioned that the considered dynamics for the labor force is quite adequate for the US statistical data in the period from 1900 to 2005. Parameter $\rho$ is small enough and equal to (approx.) $10^{-2}$.

The dynamics of the capital stock $K(t)$ is determined by the Solow model in which changes of capital depend on investment level $S(t)$ with the depreciate rate $\delta$, i.e.

$$
\dot{K}(t)=S(t)-\delta K(t) .
$$

Investments in capital constitute a part of GDP $(Y)$. Hence, it can be written as follows: $S(t)=s(t) Y(t)$, where function $s(t)$ may take any value in the range from zero to the positive constant $a_{s}$ which is less than one, i.e. $0 \leqslant s(t) \leqslant a_{s}<1$.

Changes in labor are described by the equation:

$$
\dot{L}(t)=b R(t)
$$

where function $R(t)$ denotes investments in growth of the labor efficiency. Investments $R(t)$ is also a share of GDP $(Y)$, i.e. $R(t)=r(t) Y(t)$. It is assumed that function $r(t)$ takes any values from zero to the predefined constant $a_{r}$ which is less than one. The positive parameter $b$ stands for the marginal effectiveness of investment in human capital. It is supposed that the relative useful work (per one worker) $u(t)=U(t) / P(t)$ is constant with an average value $\widetilde{u}, \quad \forall t \geq t_{0}$. Due to this assumption the production function $F[k, l, u]$ can be rewritten as follows: $F[k, l, u]=F[k, l, \widetilde{u}]=f(k, l)$.

Based on equations (11), (2) and (3), one can evaluate dynamics of relative variables $k$ and $l$.

Let functions $C(t)$ and $c(t)$ describe the total consumption level in a country and the consumption level per one worker, respectively. It is assumed that the closed economical system is considered in which $G D P(Y)$ is spent on consumption $(C)$ and investments in capital stock $(S)$ and human capital $(R)$ : $Y(t)=C(t)+S(t)+R(t)$, or in relative variables: $y(t)=c(t)+(s(t)+r(t)) y(t)$. Hence one can easily calculate consumption per one worker

$$
c(t)=(1-s(t)-r(t)) y(t) \approx(1-s(t))(1-r(t)) y(t) .
$$

Optimal Control Problem. Let us consider investments $s$ and $r$ as control variables. It is supposed that the utility function of the growth process is described by an integral consumption index discounted on the infinite horizon. We use the consumption index of the logarithmic type, rather common for the theory of endogenous growth (see [12). Let us note that the utility of such type is closely related to the notion of entropy in thermodynamics, mechanics and dynamic systems $J=\int_{t_{0}}^{+\infty} e^{-\lambda t} \ln c(t) d t$. Here, parameter $\lambda$ is the discounting factor. 
It should be mentioned that the following equality $d \ln c(t)=\frac{d c(t)}{c(t)}$ determines relative growth of the consumption $c(t)$ (4) per one worker. In fact, the introduced utility function presents the summary growth of the relative consumption adjusted to the value of money depreciation.

Problem 1. The optimal control problem presumes maximization of the utility function

$$
J=\int_{t_{0}}^{+\infty} e^{-\lambda t}(\ln (1-s(t))+\ln (1-r(t))+\ln f(k(t), l(t))) d t
$$

over trajectory $(k(\cdot), l(\cdot), s(\cdot), r(\cdot))$ of the system

$$
\left\{\begin{array}{l}
\dot{k}(t)=s(t) f(k(t), l(t))-(\delta+\rho) k(t) \\
\dot{l}(t)=b r(t) f(k(t), l(t))-\rho l(t)
\end{array}\right.
$$

with control parameters $(s(\cdot), r(\cdot))$ subject to constraints

$$
0 \leq s(t) \leq a_{s}<1, \quad 0 \leq r(t) \leq a_{r}<1, \quad 0 \leq a_{s}+a_{r}<1,
$$

and phase variables $(k(\cdot), l(\cdot))$ satisfying initial conditions $k\left(t_{0}\right)=k^{0}, l\left(t_{0}\right)=l^{0}$.

The production function $y=f(k, l)$ meets the following conditions

$P F_{1}$. For all positive values of phase variables $k$ and $l$ function $f(k, l)$ is positive with its partial derivatives, i.e. $f(k, l)>0, \quad f_{k}>0, \quad f_{l}>0$.

$P F_{2}$. For all positive values of phase variables $k$ and $l$ function $f(k, l)$ is a strictly concave function in phase variables, i.e. $f_{k k}<0, f_{k k} f_{l l}-f_{k l}^{2}>0$.

Here we use the following notations for the first and second order derivatives of the production function $f=f(k, l)$

$$
f_{k}=\frac{\partial f(k, l)}{\partial k}, f_{l}=\frac{\partial f(k, l)}{\partial l}, f_{k l}=\frac{\partial^{2} f(k, l)}{\partial k \partial l}, f_{k k}=\frac{\partial^{2} f(k, l)}{\partial k^{2}}, f_{l l}=\frac{\partial^{2} f(k, l)}{\partial l^{2}} .
$$

Let us note that the problem 1 can be solved within the optimal control theory for problems with infinite horizon (see [1, [5]).

\section{Model Analysis}

Model analysis is based on the Pontryagin maximum principle [8] for problems with infinite time horizon [1].

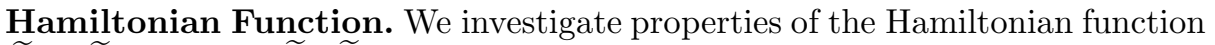
$\widetilde{H}=\widetilde{H}\left(t ; k, l ; s, r ; \widetilde{\psi}_{1}, \widetilde{\psi}_{2}\right)$ which is defined by the equality:

$$
\begin{aligned}
\widetilde{H}\left(t ; k, l ; s, r ; \widetilde{\psi}_{1}, \widetilde{\psi}_{2}\right) & =e^{-\lambda t}(\ln (1-s)+\ln (1-r)+\ln f(k, l))+ \\
& +\widetilde{\psi}_{1}(s f(k, l)-(\delta+\rho) k)+\widetilde{\psi}_{2}(\operatorname{brf}(k, l)-\rho l) .
\end{aligned}
$$

Let us formulate the main property of the Hamiltonian function (6). 
Proposition 1. The Hamiltonian function $\widetilde{H}\left(t ; k, l ; s, r ; \widetilde{\psi}_{1}, \widetilde{\psi}_{2}\right)$ is concave in control variables $s$ and $r$.

It is convenient to introduce new variables for excluding the exponential time term: $\psi_{1}=\widetilde{\psi}_{1} e^{\lambda t}, \quad \psi_{2}=\widetilde{\psi}_{2} e^{\lambda t}$ and $\widehat{H}=\widetilde{H} e^{\lambda t}$. Substituting new variables to the Hamiltonian function (6) we get the expression:

$$
\begin{aligned}
\widehat{H}\left(k, l ; s, r ; \psi_{1}, \psi_{2}\right) & =\ln (1-s)+\ln (1-r)+\ln f(k, l)+ \\
& +\psi_{1}(s f(k, l)-(\delta+\rho) k)+\psi_{2}(b r f(k, l)-\rho l) .
\end{aligned}
$$

Since control variables $s$ and $r$ satisfy to restrictions (5), the optimal control has the following structure:

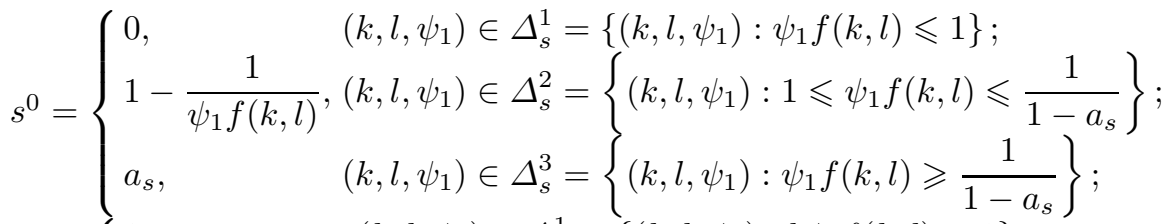

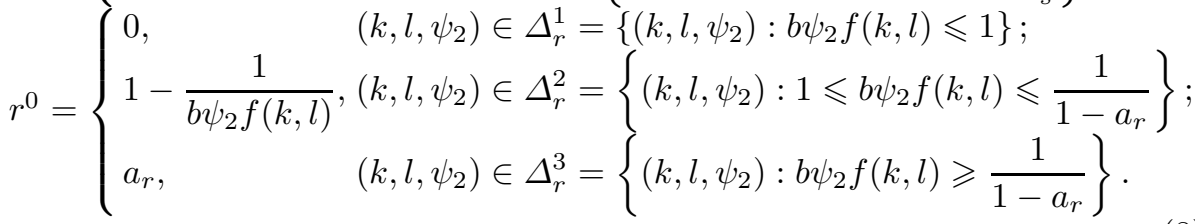

Substituting values of optimal control to the Hamiltonian function $\widehat{H}(\cdot)$ in (7) we obtain the maximized Hamiltonian: $H\left(k, l ; \psi_{1}, \psi_{2}\right)=\widehat{H}\left(k, l ; s^{0}, r^{0} ; \psi_{1}, \psi_{2}\right)$. There exist nine domains $D_{i j}=\Delta_{s}^{i} \cap \Delta_{r}^{j} \quad(i, j=1,2,3)$ of definition of the maximized Hamiltonian function. These domains are determined by the structure of optimal controls. Let us discuss important properties of the maximized Hamiltonian function.

Proposition 2. The maximized Hamiltonian function $H\left(k, l ; \psi_{1}, \psi_{2}\right)$ is a smooth function in variables $k, l$ and $\psi_{1}, \psi_{2}$ in domains $D_{i j}(i, j=\overline{1,3})$ and on boundaries between these domains.

Proposition 3. The maximized Hamiltonian is a strictly concave function in phase variables $k, l$ for all positive values of conjugate variables $\psi_{1}$ and $\psi_{2}$, if the following matrix is negatively defined:

$$
\partial f(k, l)=\left(\begin{array}{ccc}
-f & f_{k} & f_{l} \\
f_{k} & f_{k k} & f_{k l} \\
f_{l} & f_{l k} & f_{l l}
\end{array}\right), \quad \forall \quad\left(k, l, \psi_{1}, \psi_{2}\right) \in D_{22}, \psi_{1}>0, \psi_{2}>0 .
$$

Necessary and Sufficient Conditions of Optimality. Let us mention that for the control problem 1 all conditions of the existence theorem (see [1, 3]) are fulfilled. Moreover, one can formulate necessary [1] and sufficient [5] conditions of optimality for problems with infinite horizon in the form of the Pontryagin maximum principle. It should be noted that properties 2 and 3 ensure sufficiency of necessary optimality conditions [5]. 
Qualitative Analysis. Firstly, we construct the Hamiltonian system and investigate the existence of steady states. Due to the structure of the optimal control $\left(s^{0}(t), r^{0}(t)\right)$ in (8) the Hamiltonian system has different form in each domain $D_{i j} \quad(i, j=\overline{1,3})$. The special attention is given to domain $D_{22}$ with the transient control regime, where both controls are not constant.

In the domain $D_{22}=\Delta_{s}^{2} \cap \Delta_{r}^{2}$ the Hamiltonian system has the following form:

$$
\left\{\begin{aligned}
& \dot{k}=f(k, l)-(\delta+\rho) k-\frac{k}{z_{1}}=H_{1}, \\
& i=b f(k, l)-\rho l-\frac{l}{z_{2}}=H_{2}, \\
& \dot{z}_{1}=\left(\lambda-f_{k}(k, l)+\frac{f(k, l)}{k}\right) z_{1}-b \frac{k}{l} f_{k}(k, l) z_{2}+\frac{k}{f(k, l)} f_{k}(k, l)-1=H_{3}, \\
& \dot{z}_{2}=-\frac{l}{k} f_{l}(k, l) z_{1}+\left(\lambda-b f_{l}(k, l)+b \frac{f(k, l)}{l}\right) z_{2}+\frac{l}{f(k, l)} f_{l}(k, l)-1=H_{4},
\end{aligned}\right.
$$

where new adjoint variables $z_{1}$ and $z_{2}$ are defined as follows: $z_{1}=k \psi_{1}$ and $z_{2}=l \psi_{2}$ and symbols $H_{i}$ denotes functions $H_{i}=H_{i}\left(k, l, z_{1}, z_{2}\right), i=\overline{1,4}$.

Let us suppose that the Hamiltonian system has a steady state $P^{*}$ with coordinates $P^{*}=\left(k^{*}, l^{*}, z_{1}^{*}, z_{2}^{*}\right)$. In this case conjugate coordinates $z_{1}^{*}$ and $z_{2}^{*}$ of the steady state can be found from the first two equations of the Hamiltonian system, namely

$$
z_{1}^{*}=\frac{k^{*}}{f\left(k^{*}, l^{*}\right)-(\delta+\rho) k^{*}}, \quad z_{2}^{*}=\frac{l^{*}}{b f\left(k^{*}, l^{*}\right)-\rho l^{*}} .
$$

Further, we construct the linearized Hamiltonian system in a neighborhood of the steady state. Let symbol $A=\left\{\alpha_{i j}\right\}_{i, j=1}^{4}$ denotes the matrix of the linearized Hamiltonian system, where

$$
\alpha_{i 1}=\frac{\partial H_{i}\left(P^{*}\right)}{\partial k}, \alpha_{i 2}=\frac{\partial H_{i}\left(P^{*}\right)}{\partial l}, \alpha_{i 3}=\frac{\partial H_{i}\left(P^{*}\right)}{\partial z_{1}}, \alpha_{i 4}=\frac{\partial H_{i}\left(P^{*}\right)}{\partial z_{2}}, i=\overline{1,4} .
$$

\section{Nonlinear Stabilizer}

A nonlinear stabilizer is constructed under the following assumptions $A_{1}$. It is assumed that matrix $A$ has two real negative $\lambda_{1}$ and $\lambda_{2}$ and two real positive $\lambda_{3}$ and $\lambda_{4}$ eigenvalues.

This assumption means that the steady state $P^{*}=\left(k^{*}, l^{*}, z_{1}^{*}, z_{2}^{*}\right)$ has the saddle character.

Let the symbols $h_{i}=\left\{h_{i j}\right\}_{j=1}^{4}, \quad i=\overline{1,4}$ denote eigenvectors corresponding to eigenvalues $\lambda_{i}, i=\overline{1,4}$, respectively.

$A_{2}$. It is supposed that first two coordinates of eigenvectors $h_{1}$ and $h_{2}$ corresponding to negative eigenvalues $\lambda_{1}$ and $\lambda_{2}$ meet the restriction $h_{11} h_{22} \neq h_{12} h_{21}$.

Construction of Nonlinear Stabilizer. Idea of construction of the nonlinear stabilizer is based on results of the qualitative theory of differential equations 
(see 4). Namely, the trajectory of the nonlinear Hamiltonian dynamics converges to the steady state tangentially to the plane generated by eigenvectors corresponding to negative eigenvalues of the Jacobi matrix. Let us describe the algorithm of construction of the nonlinear stabilizer.

1. To build the plane $\pi$ generated by two eigenvectors $h_{1}$ and $h_{2}$ corresponding to two negative eigenvalues $\lambda_{1}$ and $\lambda_{2}$, so that the steady state $P^{*}$ belongs to this plane $\pi$.

2. To extract conjugate variables $z_{1}$ and $z_{2}$ from equations of the plane.

3 . To substitute the obtained relations of extraction instead of conjugate variables into control functions $s^{0}(t)$ and $r^{0}(t)$ corresponding to domain $D_{22}$.

As a result, the algorithm provides construction of control $\widehat{s}(t)$ and $\widehat{r}(t)$ which is called nonlinear stabilizer. Let us consider each step in details.

Plane Construction. Any vector $v$ located in the plane $\pi$ can be expressed through eigenvectors $h_{1}$ and $h_{2}$ in the following way: $v=\nu_{1} h_{1}+\nu_{2} h_{2}$. Hence, the plane $\pi$ generated by two eigenvectors $h_{1}$ and $h_{2}$ and containing the equilibrium point $P^{*}$ can be written as follows:

$$
\begin{aligned}
& k-k^{*}=\nu_{1} h_{11}+\nu_{2} h_{21}, \quad l-l^{*}=\nu_{1} h_{12}+\nu_{2} h_{22}, \\
& z_{1}-z_{1}^{*}=\nu_{1} h_{13}+\nu_{2} h_{23}, z_{2}-z_{2}^{*}=\nu_{1} h_{14}+\nu_{2} h_{24} .
\end{aligned}
$$

Due to assumption $A_{2}$ coefficients $\nu_{1}$ and $\nu_{2}$ can be found from the first two equations (11).

Extraction of Conjugate Variables. Conjugate variables $z_{1}$ and $z_{2}$ can be extracted from the equations (11) of the plane $\pi$. As a result, we obtain

$$
\begin{gathered}
z_{1}=z_{1}(k, l)=z_{1}^{*}+\gamma_{11}\left(k-k^{*}\right)+\gamma_{12}\left(l-l^{*}\right), \\
z_{2}=z_{2}(k, l)=z_{2}^{*}+\gamma_{21}\left(k-k^{*}\right)+\gamma_{22}\left(l-l^{*}\right), \\
\text { where } \gamma_{11}=-\frac{\left|\begin{array}{ll}
h_{12} & h_{13} \\
h_{22} & h_{23}
\end{array}\right|}{\left|\begin{array}{ll}
h_{11} & h_{12} \\
h_{21} & h_{22}
\end{array}\right|}, \gamma_{12}=\frac{\left|\begin{array}{ll}
h_{11} & h_{13} \\
h_{21} & h_{23}
\end{array}\right|}{\left|\begin{array}{ll}
h_{11} & h_{12} \\
h_{21} & h_{22}
\end{array}\right|}, \gamma_{21}=-\frac{\left|\begin{array}{ll}
h_{12} & h_{14} \\
h_{22} & h_{24}
\end{array}\right|}{\left|\begin{array}{ll}
h_{11} & h_{12} \\
h_{21} & h_{22}
\end{array}\right|}, \gamma_{22}=\frac{\left|\begin{array}{ll}
h_{11} & h_{14} \\
h_{21} & h_{24}
\end{array}\right|}{\left|\begin{array}{ll}
h_{11} & h_{12} \\
h_{21} & h_{22}
\end{array}\right|} .
\end{gathered}
$$

It should be mentioned that the following equalities take place

$$
z_{1}\left(k^{*}, l^{*}\right)=z_{1}^{*}, \quad z_{2}\left(k^{*}, l^{*}\right)=z_{2}^{*} .
$$

Nonlinear Stabilizer. The only thing left is to substitute expressions (12) into relations (8) for optimal controls in the domain $D_{22}$. Finally, we get the following structure of the nonlinear stabilizer:

$$
\widehat{s}(k, l)=1-\frac{k}{z_{1}(k, l) f(k, l)}, \quad \widehat{r}(k, l)=1-\frac{l}{b z_{2}(k, l) f(k, l)} .
$$

Substituting expressions for conjugate variables (12) to the first two equations of the Hamiltonian system (9) we get the stabilized Hamiltonian system:

$$
\dot{k}=f(k, l)-(\delta+\rho) k-\frac{k}{z_{1}(k, l)}, \quad i=b f(k, l)-\rho l-\frac{l}{z_{2}(k, l)} .
$$


Properties of the Nonlinear Stabilizer. Let us indicate main properties of the constructed nonlinear stabilizer.

Proposition 4. The nonlinear stabilizer (14) generates the nonlinear system (15) having the steady state with coordinates $\left(k^{*}, l^{*}\right)$ which are the same as the first two coordinates at the steady state of the original Hamiltonian system (9).

Proof of this propositions is based on the property (13) of the representation of adjoint variables $\left.z_{1}=z_{1}(k, l), z_{2}=z_{(} k, l\right)$ in the plane $\pi$ and relations (10) for conjugate coordinates $z_{1}^{*}, z_{2}^{*}$ of the steady state.

Let us consider the linearized Hamiltonian system with Jacobi matrix $A$ projected on subspace $\pi$. We substitute representation $z_{1}=z_{1}(k, l)$ and $z_{2}=z_{2}(k, l)$ (12) of conjugate variables into the first two equations of the linearized dynamics and collect similar terms

$$
\dot{k}=\bar{a}_{11}\left(k-k^{*}\right)+\bar{a}_{12}\left(l-l^{*}\right), \quad i=\bar{a}_{21}\left(k-k^{*}\right)+\bar{a}_{22}\left(l-l^{*}\right),
$$

where $\bar{a}_{i j}=\alpha_{i j}+\alpha_{13} \gamma_{1 j}+\alpha_{14} \gamma_{2 j}, i, j=1,2$.

Proposition 5. The matrix of the linearized stabilized system is the same as the matrix $\bar{A}=\left\{\bar{a}_{i j}\right\}_{i, j=1}^{2}$ of the linearized Hamiltonian system projected on plane $\pi$.

In order to prove this proposition it is necessary to linearized stabilized Hamiltonian system (15) at the steady state $\left(k^{*}, l^{*}\right)$ neighborhood.

Let the symbol $\bar{A}$ denote the matrix of the linearized stabilized system (16). The next important question deals with eigenvalues of the stabilized Hamiltonian system (15).

Proposition 6. The linearized stabilized Hamiltonian system (15) has two real negative eigenvalues coinciding with eigenvalues $\lambda_{1}$ and $\lambda_{2}$ and the following eigenvectors

$$
\bar{h}_{1}=\left(h_{11}, h_{12}\right), \quad \bar{h}_{2}=\left(h_{21}, h_{22}\right) .
$$

Proof. Basing on property 5 one can assert that the linearized stabilized Hamiltonian system coincides with the linearized Hamiltonian system (16) projected on plane $\pi$. For the Jacobi matrix $A$ evaluated at the steady state $P^{*}$ the following equalities are fulfilled $A h_{i}=\lambda_{i} h_{i}, i=\overline{1,4}$. Moreover eigenvectors $h_{1}, h_{2}$ are located at the plane $\pi$. Thus, for coordinates of these vectors are valid relations $h_{i 3}=\gamma_{11} h_{i 1}+\gamma_{12} h_{i 2}, \quad h_{i 4}=\gamma_{21} h_{i 1}+\gamma_{22} h_{i 2}, \quad i=1,2$. Using these facts let us check the following equalities $\overline{\mathcal{I}} \bar{h}_{i}=\lambda_{i} \bar{h}_{i}, i=1,2$.

$$
\begin{aligned}
& \bar{A} \bar{h}_{i}=\left(\begin{array}{ll}
\bar{a}_{11} & \bar{a}_{12} \\
\bar{a}_{21} & \bar{a}_{22}
\end{array}\right)\left(\begin{array}{l}
h_{i 1} \\
h_{i 2}
\end{array}\right)= \\
& =\left(\begin{array}{c}
\alpha_{11} h_{i 1}+\alpha_{12} h_{i 2}+\alpha_{13}\left(\gamma_{11} h_{i 1}+\gamma_{12} h_{i 2}\right)+\alpha_{14}\left(\gamma_{21} h_{i 1}+\gamma_{22} h_{i 2}\right) \\
\alpha_{21} h_{i 1}+\alpha_{22} h_{i 2}+\alpha_{23}\left(\gamma_{11} h_{i 1}+\gamma_{12} h_{i 2}\right)+\alpha_{24}\left(\gamma_{21} h_{i 1}+\gamma_{22} h_{i 2}\right)
\end{array}\right)= \\
& =\left(\begin{array}{c}
\alpha_{11} h_{i 1}+\alpha_{12} h_{i 2}+\alpha_{13} h_{i 3}+\alpha_{14} h_{i 4} \\
\alpha_{21} h_{i 1}+\alpha_{22} h_{i 2}+\alpha_{23} h_{i 3}+\alpha_{24} h_{i 4}
\end{array}\right)=\left(\begin{array}{c}
\lambda_{i} h_{i 1} \\
\lambda_{i} h_{i 2}
\end{array}\right)=\lambda_{i} \bar{h}_{i}, \quad i=1,2 .
\end{aligned}
$$


The following theorem collects all obtained results.

Theorem 1. Under assumptions $A_{1}$ and $A_{2}$ for the Hamiltonian system (9) constructed in domain $D_{22}$ and linearized in a neighborhood of the steady state $P^{*}$ the nonlinear stabilizer (14) exists and generates the nonlinear dynamical system (15) which is closed with respect to the phase variables $k, l$ and has the following properties

1. the steady state of the closed system (15) has coordinates $\left(k^{*}, l^{*}\right)$ coinciding with the phase coordinates $k$ and $l$ of the steady state $P^{*}$ of the original Hamiltonian system (9);

2. the system (15) is stabilized at the steady state $P^{*}$;

3. the eigenvectors $\bar{h}_{1}$ and $\bar{h}_{2}$ of the linearized closed system (16) generated by the nonlinear stabilizer are evaluated by formulas (17).

The proof of the theorem follows directly from properties of the nonlinear stabilizer.

Remark 1. The constructed nonlinear stabilizer generates the nonlinear system which is closed with respect to phase variables. The solution of the obtained stabilized system approximates optimal trajectories of the original Hamiltonian system in a neighborhood of the steady state, since a trajectory of the nonlinear Hamiltonian system that tends to the equilibrium point is tangent to the plane formed by two eigenvectors corresponding to negative eigenvalues. One can use this fact to estimate the growth rates of optimal trajectories The growth rates are determined by values of negative eigenvalues.

Numerical Simulations. The calculations are carried out on the basis of the data on the US economy in the period of 1900 to 2005. The data values are normalized with respect to the data values of 1900 . The production function of the Cobb-Douglas type is used: $f(k, l)=\mu k^{\alpha} l^{\beta}$. The calibration procedure for the model parameters provides the following values: $\mu=2.19942, \alpha=0.31, \beta=$ $0.09, \lambda=0.03, \delta=0.2, \rho=0.013, b=0.31, a_{s}=0.3, a_{r}=0.2, k^{0}=1, l^{0}=1$. The Hamiltonian system has the equilibrium point $P^{*}$ with coordinates $k^{*}=$ $5.75, l^{*}=5.2, z_{1}^{*}=1.8188$, and $z_{2}^{*}=2.9684$. The control parameters at the equilibrium point take the values $s^{*}=27.95$ and $r^{*}=3.79$. All four eigenvalues of the matrix $A$ calculated at the equilibrium point $P^{*}=\left(k^{*}, l^{*}, z_{1}^{*}, z_{2}^{*}\right)$ are real numbers; two of them are positive, and the other two are negative: $\lambda_{1}=-0.268, \lambda_{2}=-0.094, \lambda_{3}=0.124, \lambda_{4}=0.298$.

Trajectories of the system (15) generated by the nonlinear stabilizer (14) and the original Hamilton system (9) are calculated numerically by the Runge-Kutta method. Figure 1.(a) demonstrates phase trajectories $k(l)$ as a solutions of the stabilized (15) and Hamiltonian (9) dynamics. One can see that these trajectories almost coincide with each other especially at the vicinity of the steady state. Optimal trajectories of the capital stock $k(t)$ and labor efficiency $l(t)$ and its stabilized solutions are depicted at figures 1.(b) and 1.(c) respectively. In the steady state neighborhood optimal trajectories are very close to its stabilized solutions. 

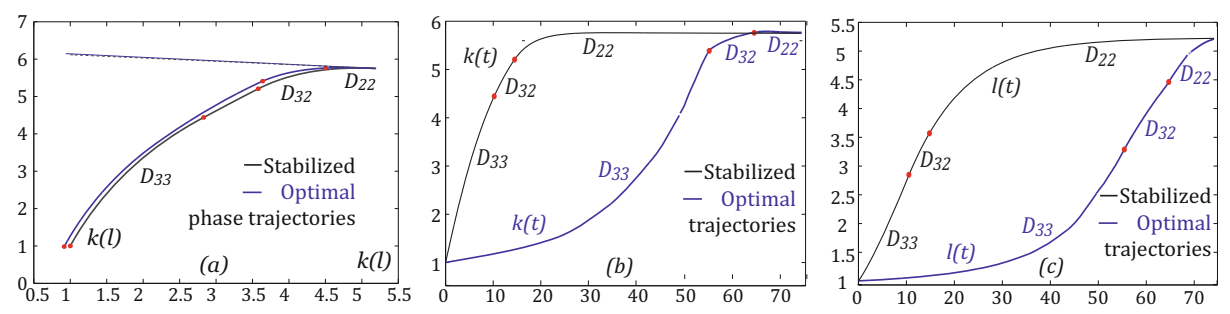

Fig. 1. Stabilized and optimal graphs (a) of phase trajectories, $k(l)$; (b) of the capital stock, $k(t)$; (c) of the labor efficiency, $l(t)$

\section{References}

1. Aseev, S.M., Kryazhimskiy, A.V.: The Pontryagin Maximum Principle and Optimal Economic Growth Problems. In: Proceedings of the Steklov Institute of Mathematics, vol. 257. Pleiades Publishing (2007)

2. Ayres, R.U., Warr, B.: The Economic Growth Engine: How Energy and Work Drive Material Prosperity. Edward Elgar Publishing, Cheltenham UK (2009)

3. Balder, E.J.: An existance result for optimal economic growth problems. J. Math. Anal. Appl. 95, 195-213 (1983)

4. Hartman, P.: Ordinary Differential Equations. J. Wiley and Sons, N.Y. (1964)

5. Krasovskii, A.A., Tarasyev, A.M.: Conjugation of Hamiltonian Systems in Optimal Control Problems. Preprints of the 17th World Congress of the International Federation of Automatic Control, IFAC, Seoul, Korea, pp. 7784-7789 (2008)

6. Krasovskii, A.N., Krasovskii, N.N.: Control under Lack of Information. Birkhauser, Boston (1995)

7. Krasovskii, N.N., Subbotin, A.I.: Game-Theoretical Control Problems. Springer, Berlin (1988)

8. Pontryagin, L.S., Boltyanskii, V.G., Gamkrelidze, R.V., Mishchenko, E.F.: The Mathematical Theory of Optimal Processes. Interscience, New York (1962)

9. Sanderson, W.: The SEDIM Model: Version 0.1. IIASA Interim Report IR-04-041, 42 pages (2004)

10. Shell, K.: Applications of Pontryagin's Maximum Principle to Economics. Mathematical Systems Theory and Economics 1, 241-292 (1969)

11. Solow, R.M.: Growth Theory: An Exposition. Oxford University Press, New York (1970)

12. Tarasyev, A.M., Watanabe, C.: Optimal Dynamics of Innovation in Models of Economic Growth. Journal of Optimization Theory and Applications 108, 175-203 (2001) 